\title{
Noise estimation of beam position monitors at RHIC
}

\author{
X. Shen, S.Y. Lee
}

Indiana University

\author{
M. Bai \\ Brookhaven National Laboratory
}

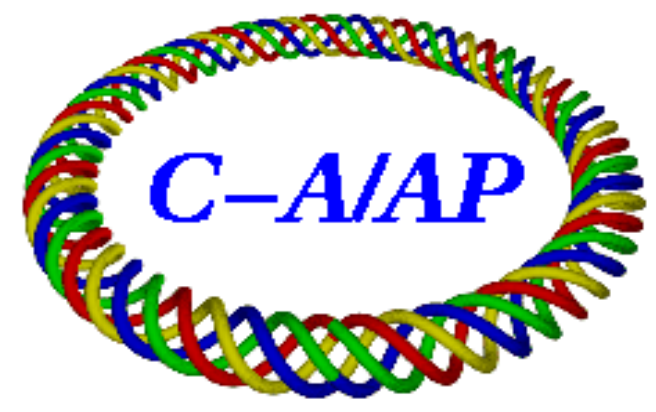

\section{Collider-Accelerator Department Brookhaven National Laboratory Upton, NY 11973}

Notice: This document has been authorized by employees of Brookhaven Science Associates, LLC under Contract No. DE-AC02-98CH10886 with the U.S. Department of Energy. The United States Government retains a nonexclusive, paid-up, irrevocable, world-wide license to publish or reproduce the published form of this document, or allow others to do so, for United States Government purposes. 


\title{
Noise estimation of beam position monitors at RHIC
}

\author{
X. Shen, ${ }^{1}$ M. Bai, ${ }^{2}$ and S. Y. Lee ${ }^{1}$ \\ 1) Department of Physics, Indiana University, Bloomington, \\ IN 47405 \\ 2) Brookhaven National Laboratory, Upton, NY, 11973, USA
}

A detailed noise analysis of beam position monitors (BPMs) at the Relativistic Hadron Ion Collider (RHIC) plays an important role in any turn-by-turn analysis. An independent component analysis (ICA) algorithm is proven by simulation to be efficient in BPM noise estimation. Application of ICA algorithm to experimental turn-by-turn data reveals the distribution of BPM noise level, which is consistent with the characteristics of RHIC BPMs.

PACS numbers: $29.90+\mathrm{r}$

\section{INTRODUCTION}

Beam position monitors (BPM) are used to record the average orbits and transverse turnby-turn displacements of the beam centroid motion. At the Relativistic Hadron Ion Collider (RHIC), there are 160 BPMs for each plane in each of the Blue and Yellow rings: 72 dualplane BPMs in the insertion regions (IR) and 176 single-plane modules in the arcs. Each BPM is able to acquire 1024 or 4096 consecutive turn-by-turn beam positions. The acquired BPM turn-by-turn data consist of linear superposition of narrow-band beam motions, each of which has its characteristic frequency, e.g., betatron oscillation, synchrotron oscillations, and non-linear oscillations. Inevitably, there are broadband noisy signals in the turn-by-turn data due to BPM electronics as well as other sources. Sometimes, a few BPMs may even be malfunctioning. Singular value decomposition (SVD) has been used to identify the faulty BPMs from turn-by-turn data ${ }^{1}$. However, a detailed study of the BPM noise performance is critical for reliable optics measurement and beam dynamics analysis based on turn-by-turn data.

Independent component analysis (ICA) is a powerful blind source separation method ${ }^{2}$. With its high efficiency in separating narrow-band source signals from sampled data, ICA has been utilized to extract characteristic beam motion signals from turn-by-turn data for 
beam optics measurement at Fermilab Booster ${ }^{3}$, Los Alamos Proton Storage Ring ${ }^{4}$, and RHIC $^{5}$. Compared to the SVD-based principle component analysis ${ }^{6}$, ICA is more robust in the presence of noise ${ }^{3}$. Hence, ICA is applied to turn-by-turn data driven by AC dipoles ${ }^{7}$ for optics measurement and correction ${ }^{5}$. This paper uses ICA to estimate RHIC BPM noise.

We organize our paper as follows. A brief introduction of the ICA technique for BPM noise estimation is presented in Section II. Section III presents simulation results of ICA performance on BPM noise estimation. Estimated RHIC BPM noise by application of ICA to experimental data is presented in Sec. IV.

\section{ICA ALGORITHM FOR BPM NOISE ESTIMATION}

The model of ICA assumes that in a complex dynamical system an $m$-dimensional observable vector $\mathbf{X}(t)$ is related to an $n$-dimensional source signal vector $\mathbf{s}(t)$ as:

$$
\mathbf{X}(t)=\mathbf{A s}(t)+\mathbf{N}(t),
$$

where the $m \times n(m \geq n)$ matrix $\mathbf{A}$ is called mixing matrix and $\mathbf{N}(t)$ is the noise vector which is assumed to be stationary, temporally white and statistically independent of source signal $\mathbf{s}(t)$. The goal of ICA is to determine the mixing matrix $\mathbf{A}$ and the source signals $\mathbf{s}(t)$ from the sampled observable vector $\mathbf{X}(t)$ without a priori knowledge of the underlying physical process. The source signals $\mathbf{s}_{i}$ are assumed to be mutually independent and temporally uncorrelated. The time-lagged covariance matrix $\mathbf{C}_{\mathbf{s}}(\tau)=\left\langle\mathbf{s}(t) \mathbf{s}(t+\tau)^{T}\right\rangle$ is therefore diagonal $^{8}$, i.e., $\left\langle\mathbf{s}_{i}(t) \mathbf{s}_{j}(t+\tau)^{T}\right\rangle=S_{i}(\tau) \delta_{i, j}$, where the superscript $T$ means a transpose, $\tau$ is a time-lag constant and the average is taken over time. From Eq.(1) the relationship between the time-lagged covariance matrix $\mathbf{C}_{\mathbf{X}}(\tau)=\left\langle\mathbf{X}(t) \mathbf{X}(t+\tau)^{T}\right\rangle$ and $\mathbf{C}_{\mathbf{s}}(\tau)$ is obtained:

$$
\begin{aligned}
& \mathbf{C}_{\mathbf{X}}(0)=\mathbf{A C}_{\mathbf{s}}(0) \mathbf{A}^{T}+\operatorname{diag}\left(\sigma_{1}^{2}, \sigma_{2}^{2}, \ldots, \sigma_{M}^{2}\right), \\
& \mathbf{C}_{\mathbf{X}}(\tau)=\mathbf{A C}_{\mathbf{s}}(\tau) \mathbf{A}^{T}, \tau \neq 0,
\end{aligned}
$$

where $\operatorname{diag}(\cdots)$ denotes a diagonal matrix with diagonal elements given in the parenthesis, $\sigma_{i}$ is the standard deviation, which is also denoted as noise level in this paper, of random noise at the $i$-th BPM. Since $\mathbf{C}_{\mathbf{s}}(\tau)$ is diagonal, the mixing matrix $\mathbf{A}$ is found as the joint diagonalizer of $\mathbf{C}_{\mathbf{X}}(\tau)$. In the following, the algorithms of ICA to extract the mixing matrix A and source signals $\mathbf{s}$ are discussed. 
First, a whitening procedure is applied to preprocess the raw data. The zero time-lagged covariance matrix $\mathbf{C}_{\mathbf{X}}(0)$ is decomposed by singular valued decomposition (SVD):

$$
\mathbf{C}_{\mathbf{X}}(0)=\left(\mathbf{U}_{1}, \mathbf{U}_{2}\right)\left(\begin{array}{cc}
\boldsymbol{\Lambda}_{1} & 0 \\
0 & \boldsymbol{\Lambda}_{2}
\end{array}\right)\left(\begin{array}{c}
\mathbf{U}_{1}^{T} \\
\mathbf{U}_{2}^{T}
\end{array}\right)
$$

where $\mathbf{U}_{1}$ and $\mathbf{U}_{2}$ are orthogonal matrices, $\boldsymbol{\Lambda}_{1}$ and $\boldsymbol{\Lambda}_{2}$ are diagonal matrices with $\min \left(\operatorname{diag}\left[\boldsymbol{\Lambda}_{1}\right]\right) \geq$ $\lambda_{c}>\max \left(\operatorname{diag}\left[\boldsymbol{\Lambda}_{2}\right]\right) \geq 0, \lambda_{c}$ is a threshold to remove the singularity of the covariance matrix, and the elements of the $l \times l(l \leq n)$ diagonal matrix $\Lambda_{1}$ are arranged in a descending manner $\lambda_{1} \geq \lambda_{2} \geq \cdots \geq \lambda_{l}$. Specifically, we denote $n_{c}=l$ as the cut-off number. Using the matrix

$$
\mathbf{V} \equiv \boldsymbol{\Lambda}_{1}^{-1 / 2} \mathbf{U}_{1}^{T}
$$

an $l$-dimensional vector $\mathbf{Z}=\mathbf{V X}$ is constructed. $\mathbf{Z}$ is spatially white because it satisfies $\left\langle\mathbf{Z Z}^{T}\right\rangle=\mathbf{I}$, where $\mathbf{I}$ is the $l \times l$ identity matrix. The whitening procedure removes redundant information and noise from the raw data and de-correlates and normalizes the data to facilitate the next step.

Using the whitened data $\mathbf{Z}$ and a set of time-lag constants $\left\{\tau_{k}\right\}(k=1,2, \ldots, K)$, the timelagged covariance matrices are computed as $\left\{\mathbf{C}_{\mathbf{Z}}\left(\tau_{k}\right)=\left\langle\mathbf{Z}(t) \mathbf{Z}\left(t+\tau_{k}\right)\right\rangle\right\}$. The symmetrized time-lagged covariance matrices are formed as $\overline{\mathbf{C}}_{\mathbf{Z}}\left(\tau_{k}\right)=\left[\mathbf{C}_{\mathbf{Z}}\left(\tau_{k}\right)+\mathbf{C}_{\mathbf{Z}}^{T}\left(\tau_{k}\right)\right] / 2$ such that they are real and symmetric and thus their eigenvalue decompositions are well defined. At last, a Jacobi-like algorithm ${ }^{9}$ is applied to find a unitary matrix $\mathbf{W}$ to jointly diagonalize $\overline{\mathbf{C}}_{\mathbf{Z}}\left(\tau_{k}\right)$ such that $\overline{\mathbf{C}}_{\mathbf{Z}}\left(\tau_{k}\right)=\mathbf{W} \mathbf{D}_{k} \mathbf{W}^{T}$, where $\mathbf{D}_{k}$ 's are diagonal matrices. The source signals are given by $\mathbf{s}=\mathbf{W}^{T} \mathbf{V X}$ and the mixing matrix by $\mathbf{A}=\mathbf{V}^{-1} \mathbf{W}$.

The turn-by-turn BPM data is normally composed of beam motions driven by different physical sources with their own characteristic frequencies, such as betatron oscillation, synchrotron oscillation and electronic noises. Separation of the BPM data into these individual beam motion is of great interest for deeper understanding of beam dynamics. The source signals separated by ICA have non-overlapping power spectra because of their properties of mutual independence. Therefore ICA is particularly suitable for the task of identifying individual beam motion. 
To apply ICA, we organize the data matrix as

$$
\mathbf{X}=\left(\begin{array}{cccc}
x_{1}(1) & x_{1}(2) & \ldots & x_{1}(P) \\
x_{2}(1) & x_{2}(2) & \ldots & x_{2}(P) \\
\vdots & \vdots & \ddots & \vdots \\
x_{M}(1) & x_{M}(2) & \ldots & x_{M}(P)
\end{array}\right)
$$

where $x_{i}(j)$ is the reading of the $i$-th BPM at the $j$-th turn, and the DC component has been removed from each BPM channel. ICA algorithm is used to extract the source signal $\mathbf{s}$ and mixing matrix $\mathbf{A}$ in Eq.(1). $\mathbf{s}$ and $\mathbf{A}$ are also known as temporal function and spatial function, respectively. The combination of the $i$-th row of $\mathbf{s}, \mathbf{s}_{i}$, and the $i$-th column of $\mathbf{A}, \mathbf{A}_{i}$ is defined as the $i$-th mode. The correspondence of the $i$-th mode to a certain beam motion is identified by comparing the frequency of the temporal function $\mathbf{s}_{i}$ to the characteristic frequency of the beam motion. Signal of characteristic beam motion can then by extracted by combining the temporal and spatial functions. For example, the betatron oscillation component $\mathbf{X}_{B}(t)$ of BPM data involves two modes with identical frequency spectra

$$
\mathbf{X}_{B}(t)=\mathbf{A}_{b 1} s_{b 1}(t)+\mathbf{A}_{b 2} s_{b 2}(t),
$$

where $\mathbf{A}_{f 1}$ and $\mathbf{A}_{f 2}$ are the spatial functions, and $s_{f 1}(t)$ and $s_{f 2}(t)$ are the cosine-like and sine-like source signals, respectively.

Other components of beam characteristic oscillation can be extracted by combining the corresponding temporal and spatial functions in a similar way. The estimated BPM noise signals $\hat{\mathbf{N}}$ is then given by

$$
\hat{\mathbf{N}}=\mathbf{X}-\sum \mathbf{X}_{\text {beam }},
$$

where $\sum \mathbf{X}_{\text {beam }}$ is a sum of signals of characteristic beam oscillations. Finally, the covariance matrix $\mathbf{C}_{\hat{\mathbf{N}}}$ provides an estimation of noise levels $\hat{\sigma}_{i} \operatorname{via} \mathbf{C}_{\hat{\mathbf{N}}}=\left\langle\hat{\mathbf{N}} \hat{\mathbf{N}}^{T}\right\rangle=\operatorname{diag}\left(\hat{\sigma}_{1}^{2}, \hat{\sigma}_{2}^{2}, \ldots, \hat{\sigma}_{M}^{2}\right)$.

\section{SIMULATION OF ICA ESTIMATION OF BPM NOISE}

In order to evaluate the capability of the proposed algorithm in actual data analysis, ICA was employed to process turn-by-turn data generated by tracking simulations. In one simulation, a single on-momentum particle initially rested at the origin of phase space was driven by an ac dipole for 3072 turns. The ac dipole kick amplitude was linearly ramped up 
to its maximum value during the first 1024 turns. It was then kept constant for the second 1024 turns, and linearly ramped down during the last 1024 turns. The maximum ac dipole kick amplitude was chosen to produce a coherent oscillation amplitude about $300 \mu \mathrm{m}$ in the middle of an arc, similar to the level of driven oscillation amplitude as observed during experiment. The beam position data was recorded during the second 1024 turns at all available BPMs. To simulate the additive noise signals in Eq. (1), random numbers subjected to Gaussian distribution with zero mean and chosen standard deviation were assigned to the BPMs, in which each set of random numbers has a unique random seed. The standard deviation of noise assigned to each BPM was drawn from a real uniform random distribution in a range between 0 and $120 \mu \mathrm{m}$, which is an estimation of the maximum rms noise level. To obtain statistical estimations, the simulation was repeated for 10 times, in which different random seeds were regenerated for each time.

ICA was then applied to the simulated turn-by-turn data. Figure 1 shows a typical distribution of singular values for the simulation data, in which the first two mode dominate the others by more than 3 orders of magnitude. Figure 2 shows the spectrum of temporal functions from Mode 1 to 4 , in which mode 1 and 2 are identified as physical modes corresponding to the ac dipole driven oscillation, while mode 3 and 4 are mostly dominated by white noise. In our study, the cut-off number $n_{c}$ is chosen as the mode number before the first non-physical mode. Therefore, in this case $n_{c}=2$. The estimated noise levels $\sigma_{\text {estimated }}$ are shown in Fig. 3 on top of the noise levels set in the simulation $\sigma_{\text {set. }}$. Within the range of error bars, $\sigma_{\text {estimated }}$ is in good agreement with $\sigma_{\text {set }}$, which validates the ICA algorithm.

\section{RHIC BPM NOISE ESTIMATION WITH EXPERIMENTAL DATA}

During the 2013 RHIC polarized proton run, experiments of applying ICA to ac dipole based optics measurement and correction were conducted ${ }^{5}$. The turn-by-turn data taken during the experiments were analyzed by the ICA algorithm for estimation of BPM noise levels. Typical singular value distribution is shown in Fig. 4. An example of the spectrum of these first 8 modes are shown in Fig. 5. Singular values of the first two modes, which are corresponding to the ac dipole driven oscillation, dominate the others by more than 3 orders of magnitude. Singular value of mode 3 shows characteristic frequency of synchrotron oscillation. For all turn-by-turn data, the first 3 modes exhibit the same characteristic 


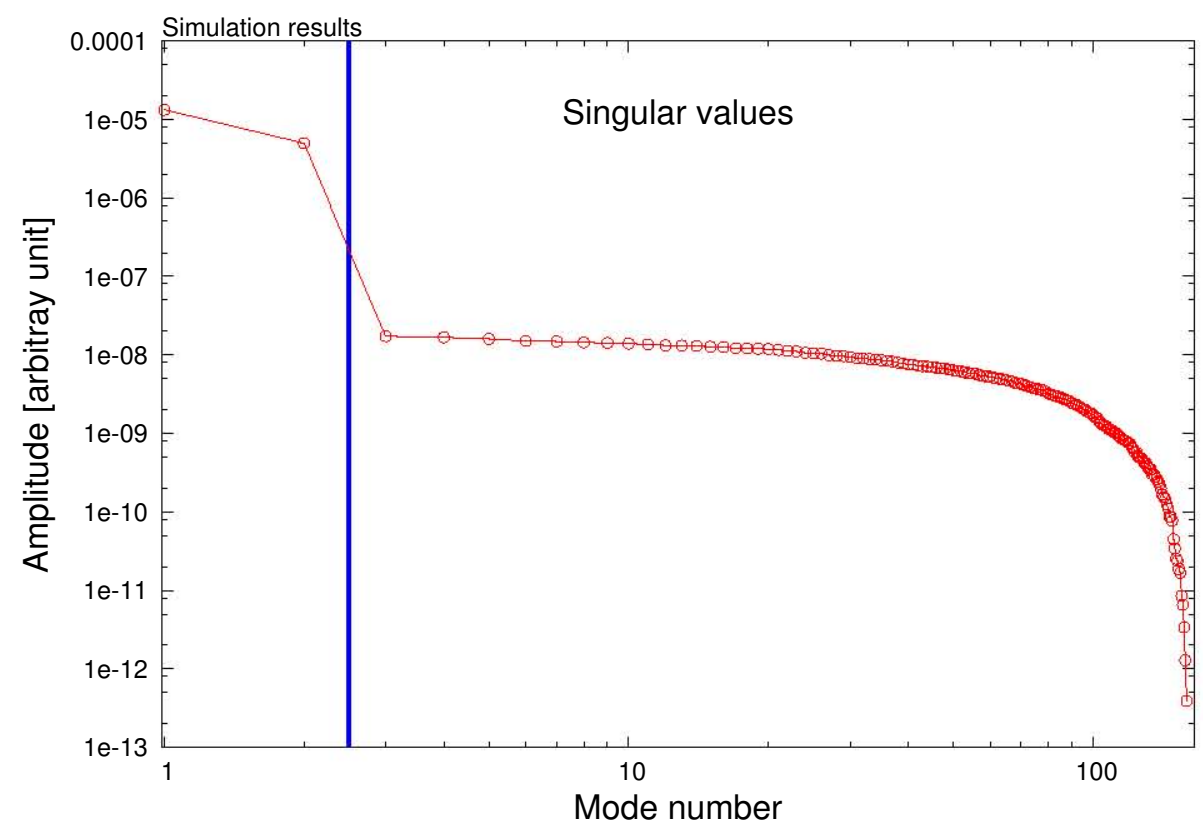

FIG. 1. Typical distribution of singular values for the simulated turn-by-turn data. The cut-off mode number is chosen to be $n_{c}=2$.
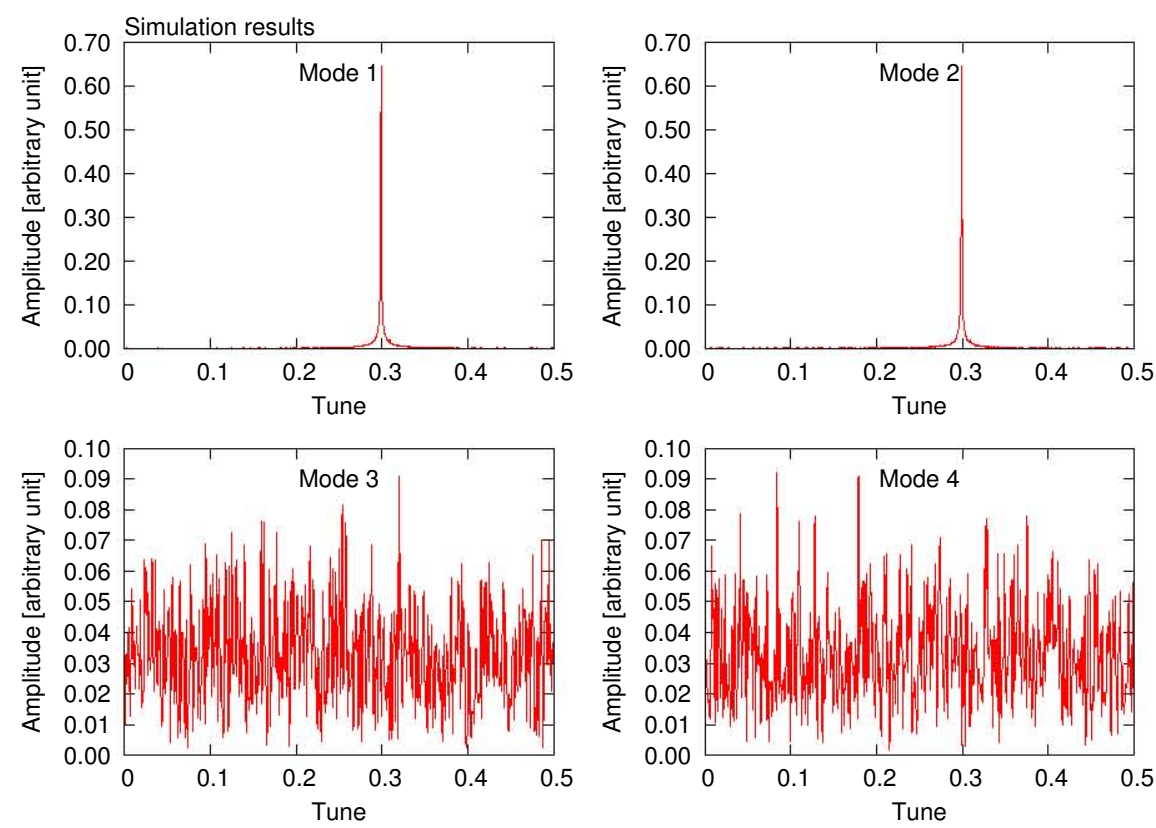

FIG. 2. Typical spectrum of temporal functions from Mode 1 to 4 for the simulated turn-by-turn data. Mode 1 and 2 exhibit dominant ac dipole driven frequency, while mode 3 and 4 show the characteristics of white noise.

frequencies which are similar to the first 3 modes shown in Fig. 5 . For the other modes, some of them are dominated by white noise, while the others have some peaks of characteristic frequencies with smaller amplitudes on top of a white noise background. An example is 


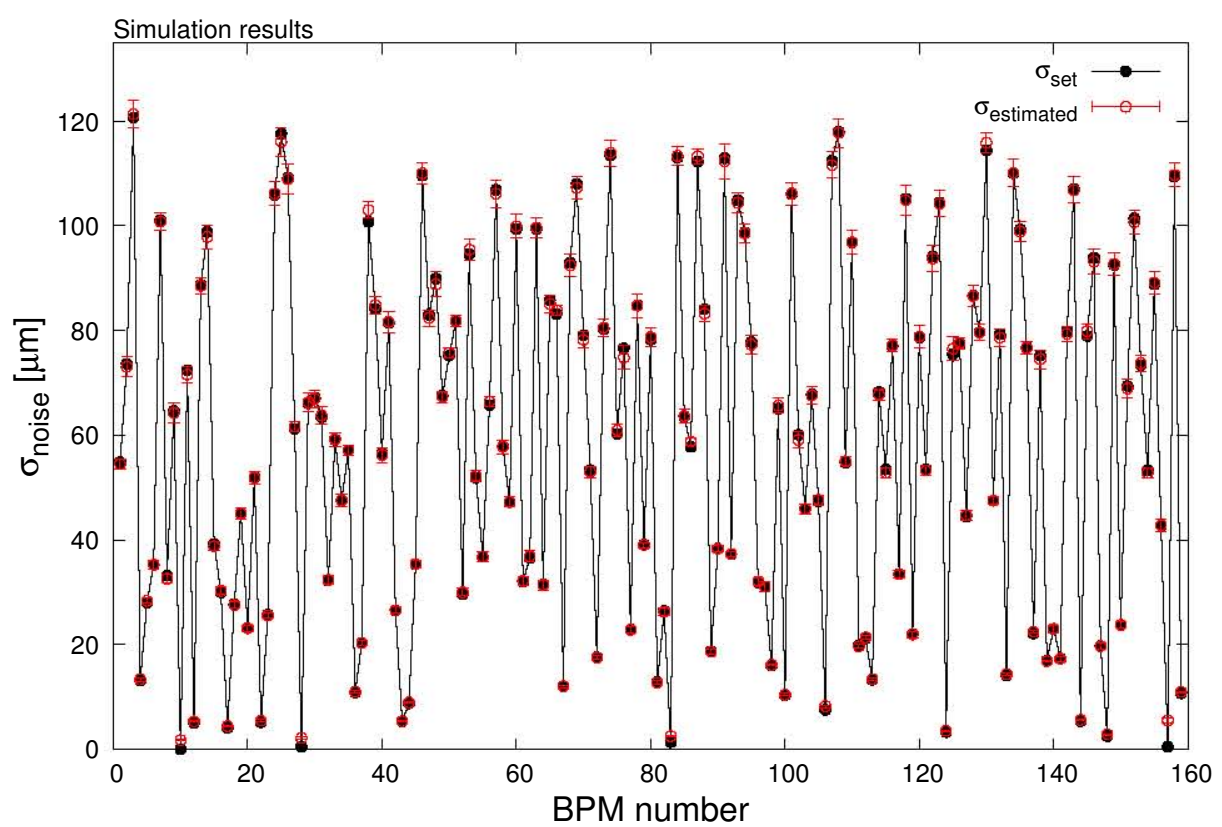

FIG. 3. Comparison of noise levels set by the simulation $\sigma_{\text {set }}$ and those estimated by the ICA algorithm $\sigma_{\text {estimated }}$ For each BPM, $\sigma_{\text {estimated }}$ is calculated as the average of a total of 10 estimations in which, for each time, the white noise added to each BPM was generated by a unique random seed. The error bars reflect the standard deviation.

shown in mode 4 to 8 in Fig. 5. However, the relative sequence of mode 4 to 8 can vary for different turn-by-turn data, because the singular values of these modes are very close in magnitude. These facts show that there are still weak physical signals in modes other than the first 3 important modes. As it is shown hereinafter, a choice of large cut-off mode number $n_{c}$ may results in including additional noise signals in the term $\sum \mathbf{X}_{\text {beam }}$ of Eq. (8), in which sense the noise $\hat{\mathbf{N}}$ is underestimated.

For example, the estimated noise levels for BPMs of the Blue and Yellow rings are shown in Fig. 6 and 8, respectively. Histograms of noise level distribution for BPMs of the Blue and Yellow rings are shown in Fig. 7 and 9, correspondingly. The two estimations with $n_{c}=4$ and $n_{c}=6$ are generally the same, except at a few triplet BPMs. In addition, the difference between the two estimations does not show a pattern related to any realistic beam motions. Hence, the histograms of noise level distribution are only calculated for the case with $n_{c}=4$. The overall noise level for both rings after the beta-beat correction is large than the one before the correction. The difference of the overall noise level before and after correction may have been resulted from the change of beam intensity and beam size after the beta-beat correction, as is shown in Table I, and that the systematic errors of RHIC BPM 


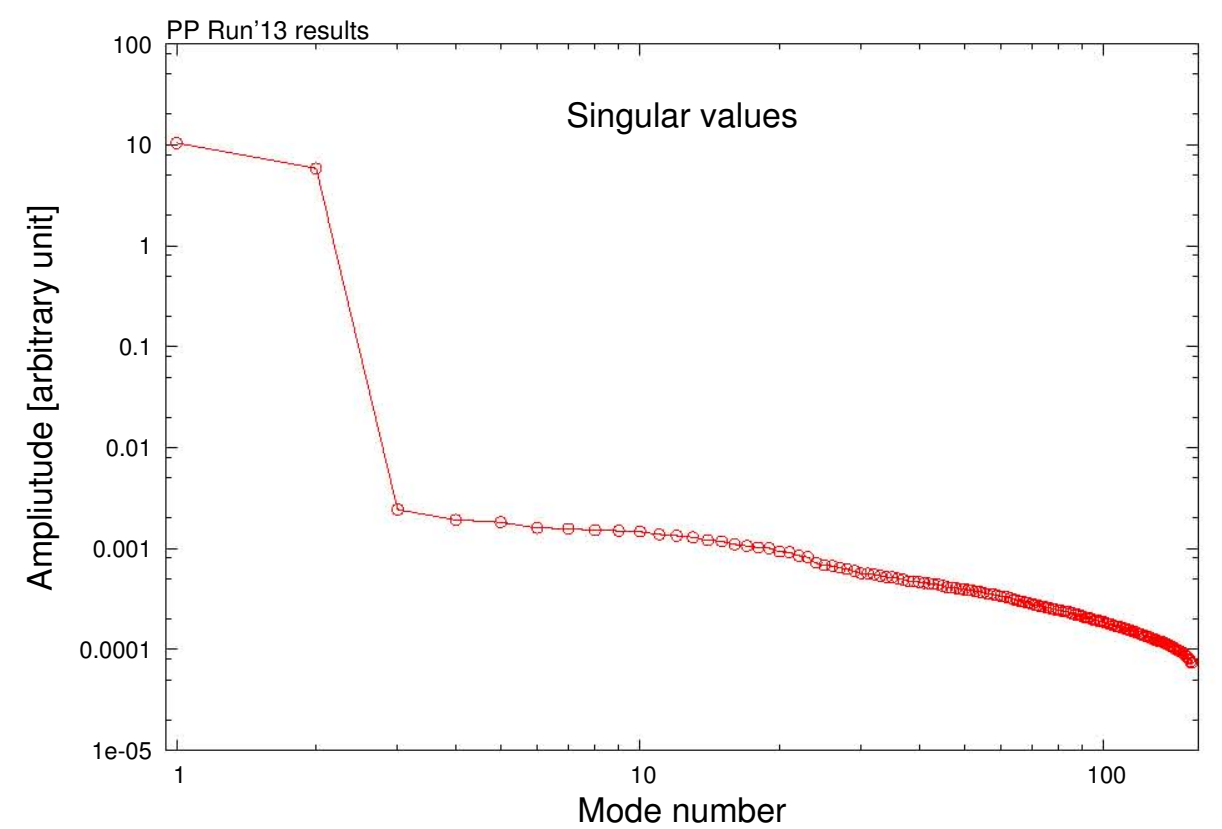

FIG. 4. Typical distribution of singular values for experimental turn-by-turn data.
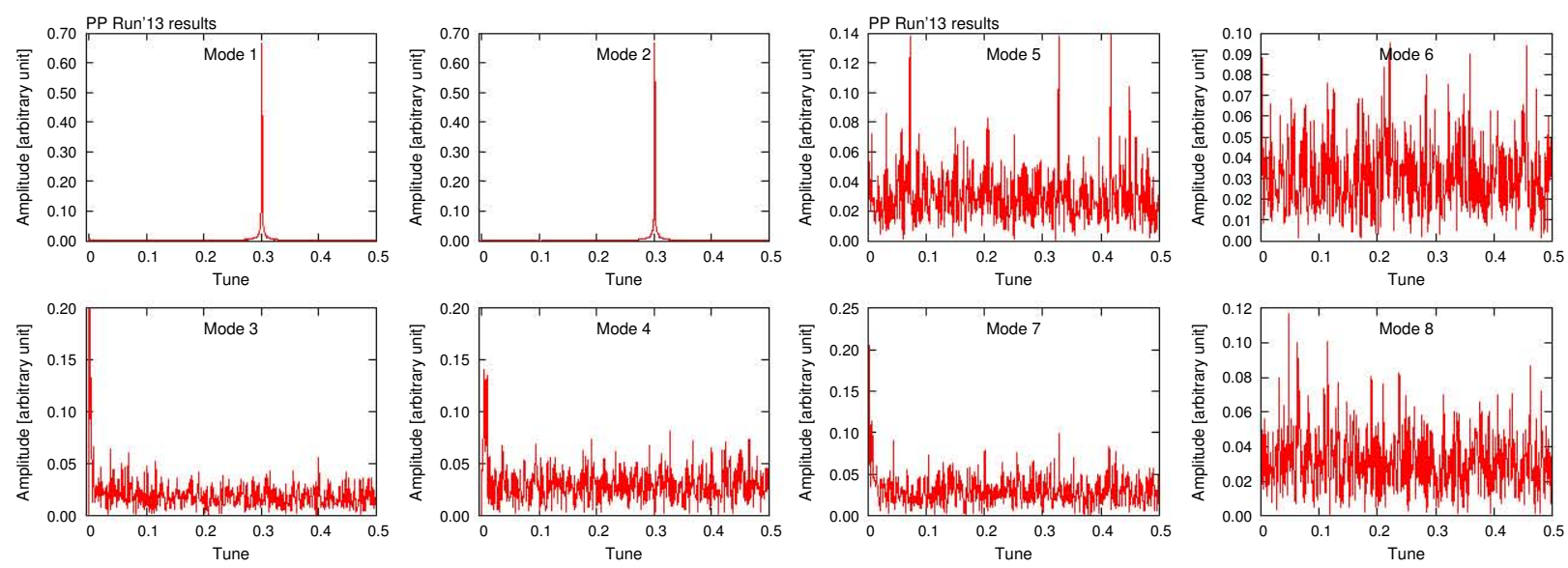

FIG. 5. Typical spectrum of temporal functions from Mode 1 to 8 for the turn-by-turn data of 2013 polarized proton run Fill\#17420.

readings increase as beam intensity decreases ${ }^{11}$. There are 4 types of BPMs at RHIC, listed in Table II. Different types of BPMs show different noise levels. The noise level distribution is roughly: $\sigma_{\text {type } 1} \approx \sigma_{\text {type } 2}<\sigma_{\text {type } 4}<\sigma_{\text {type } 3}<65 \mu \mathrm{m}$, as is shown in Fig. 6 and 8 . This fact may have resulted from the characteristics of each type of BPM, e.g., aperture, number of planes, and warm/cold working condition. Particularly in an arc, the noise level is larger as the BPM is closer to the middle of the arc. Further investigations are needed to identify the sources of this noise level distribution.

For comparison, same analysis was conducted to turn-by-turn data of UU experiments in 

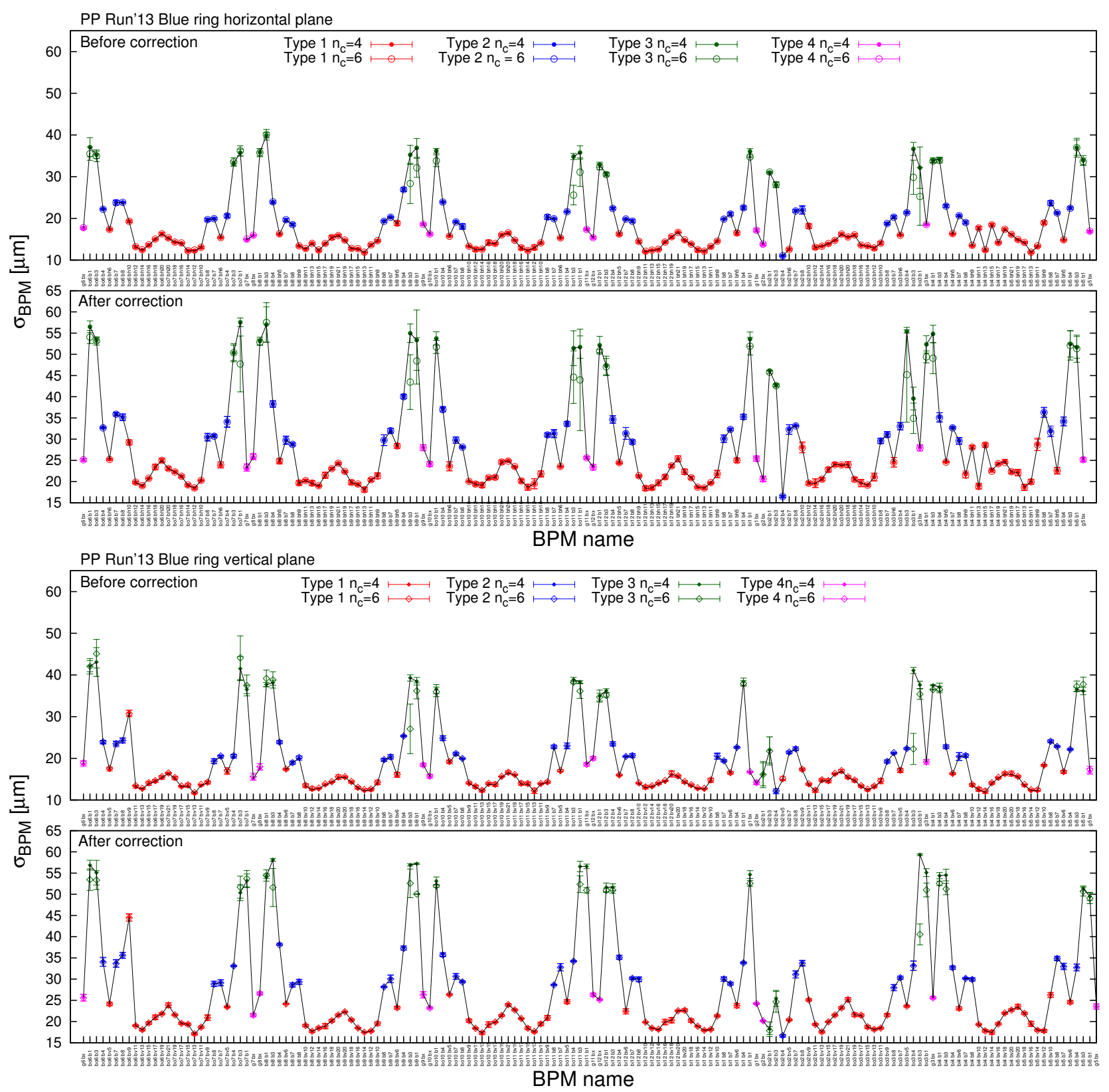

FIG. 6. Estimated noise levels of BPMs in the horizontal (top) and vertical (bottom) plane for the Blue ring of PP Run'13 Fill\#17420 for $n_{c}=4$ (solid points) and $n_{c}=6$ (hollow points). The case with $n_{c}=6$ underestimates the noise at some triplet BPMs.

Run 2012. Figure 10 and 12 show the estimated noise levels for the Blue and Yellow rings, respectively. The noises at a few DX BPMs are underestimated by the case with $n_{c}=6$. Histograms of noise level distribution for BPMs of the Blue and Yellow rings with $n_{c}=4$ are shown in Fig. 11 and 13, respectively. In this situation, noise distribution is overall: $\sigma_{\text {type } 1}<\sigma_{\text {type } 2}<\sigma_{\text {type } 3}<\sigma_{\text {type } 4}<110 \mu \mathrm{m}$. The reason for the large noise level in type 

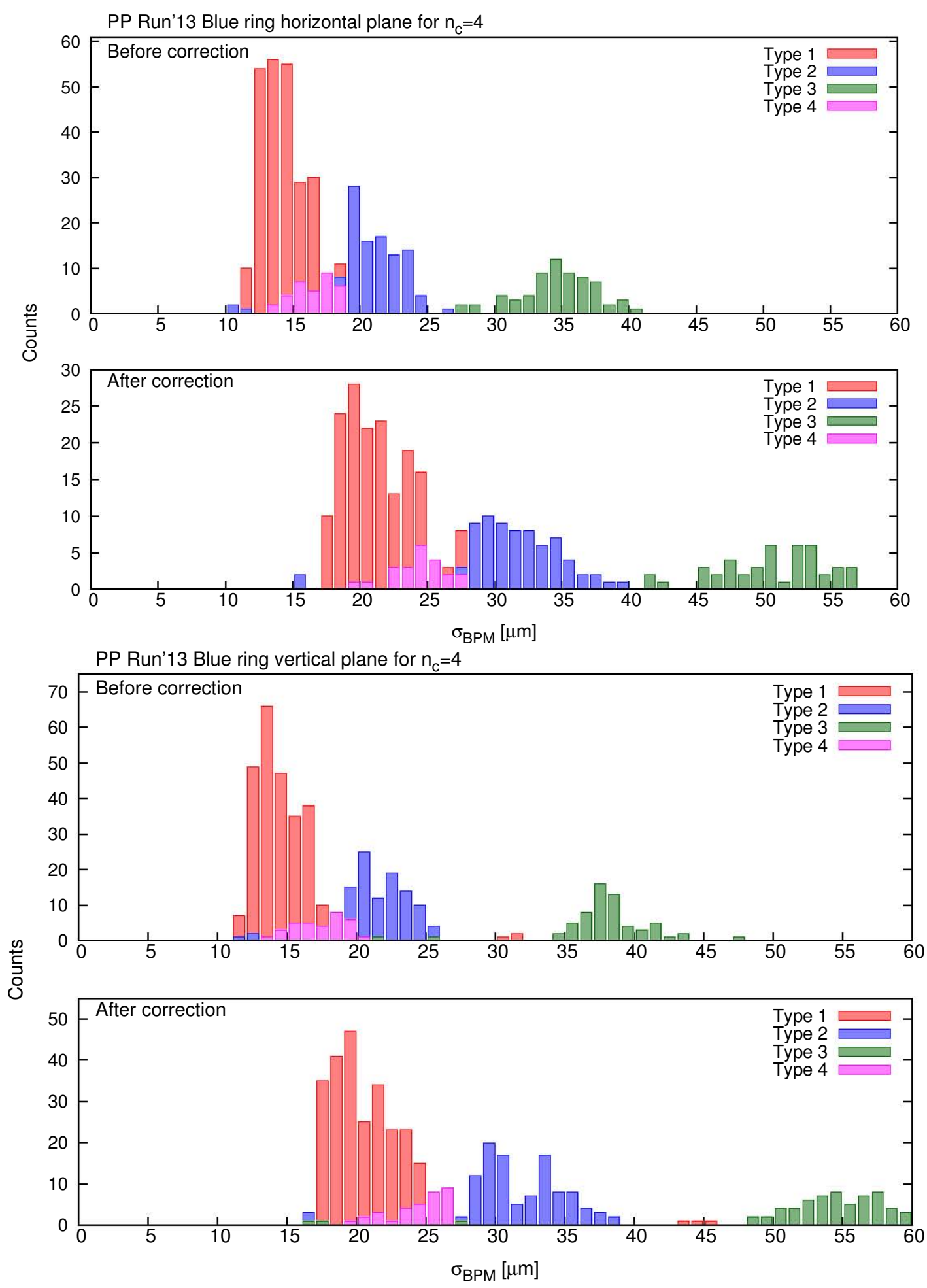

FIG. 7. Histogram of estimated noise levels of BPMs in the horizontal (top) and vertical (bottom) plane for the Blue ring of PP Run'13 Fill\#17420 for $n_{c}=4$. The overall noise level becomes larger after the beta-beat correction. Roughly in each plot, $\sigma_{\text {type } 1} \approx \sigma_{\text {type } 2}<\sigma_{\text {type } 4}<\sigma_{\text {type } 3}<60 \mu$ m. 

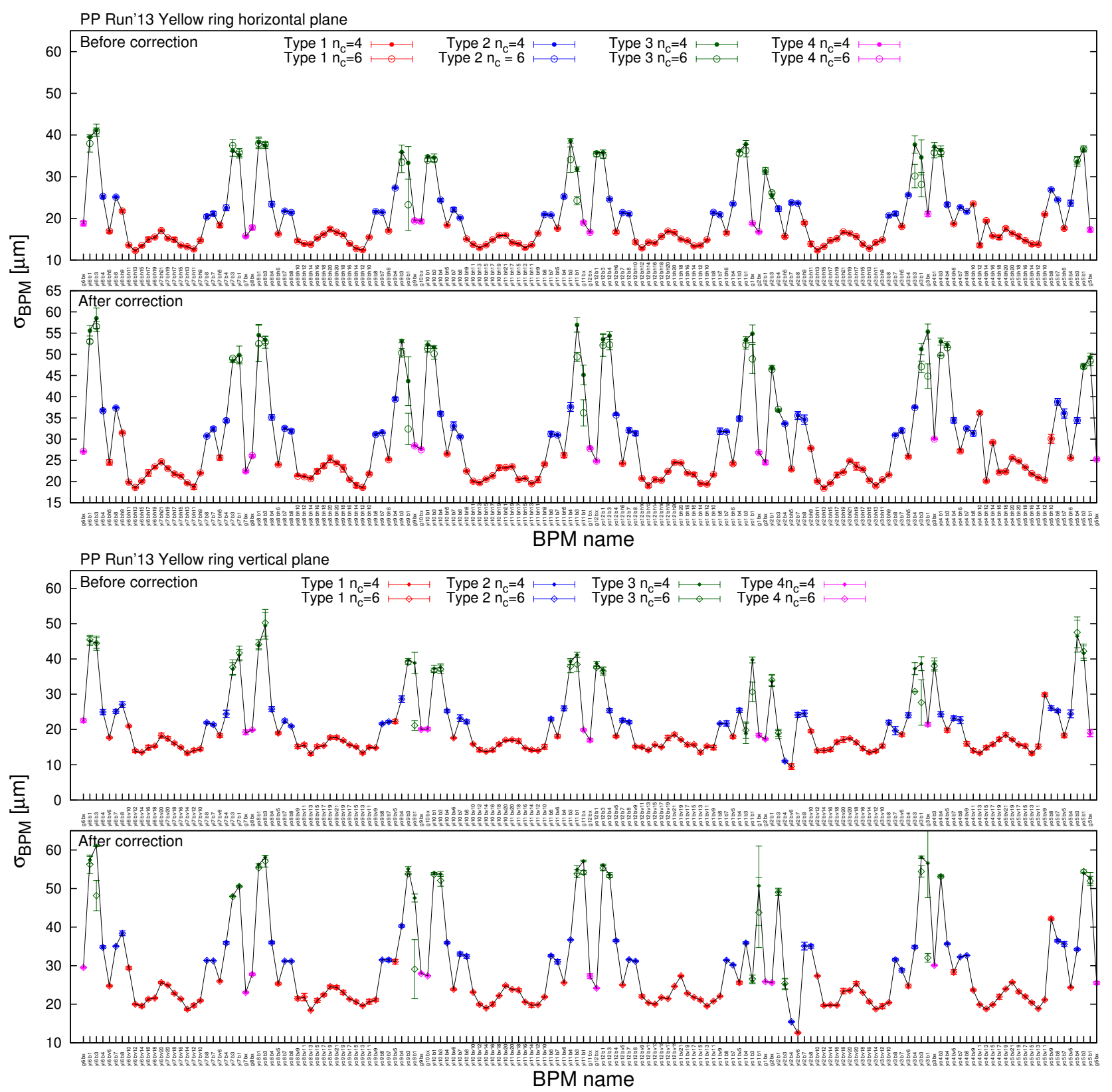

FIG. 8. Estimated noise levels of BPMs in the horizontal (top) and vertical (bottom) plane for the Yellow ring of PP Run'13 Fill\#17420 for $n_{c}=4$ (solid points) and $n_{c}=6$ (hollow points). The case with $n_{c}=6$ underestimates the noise at some triplet BPMs.

4 BPMs, which are also the DX BPMs, may have resulted from the fact that DX BPMs are common to both beams so that their signals are more sensitive to timing issues, etc. Similarly, overall noise level after correction is larger than the one before correction, which may be correlated with larger beam size after correction, as shown in Table III. The growth of beam emittance after correction was largely due to the non-adiabatic ac dipole excitation 

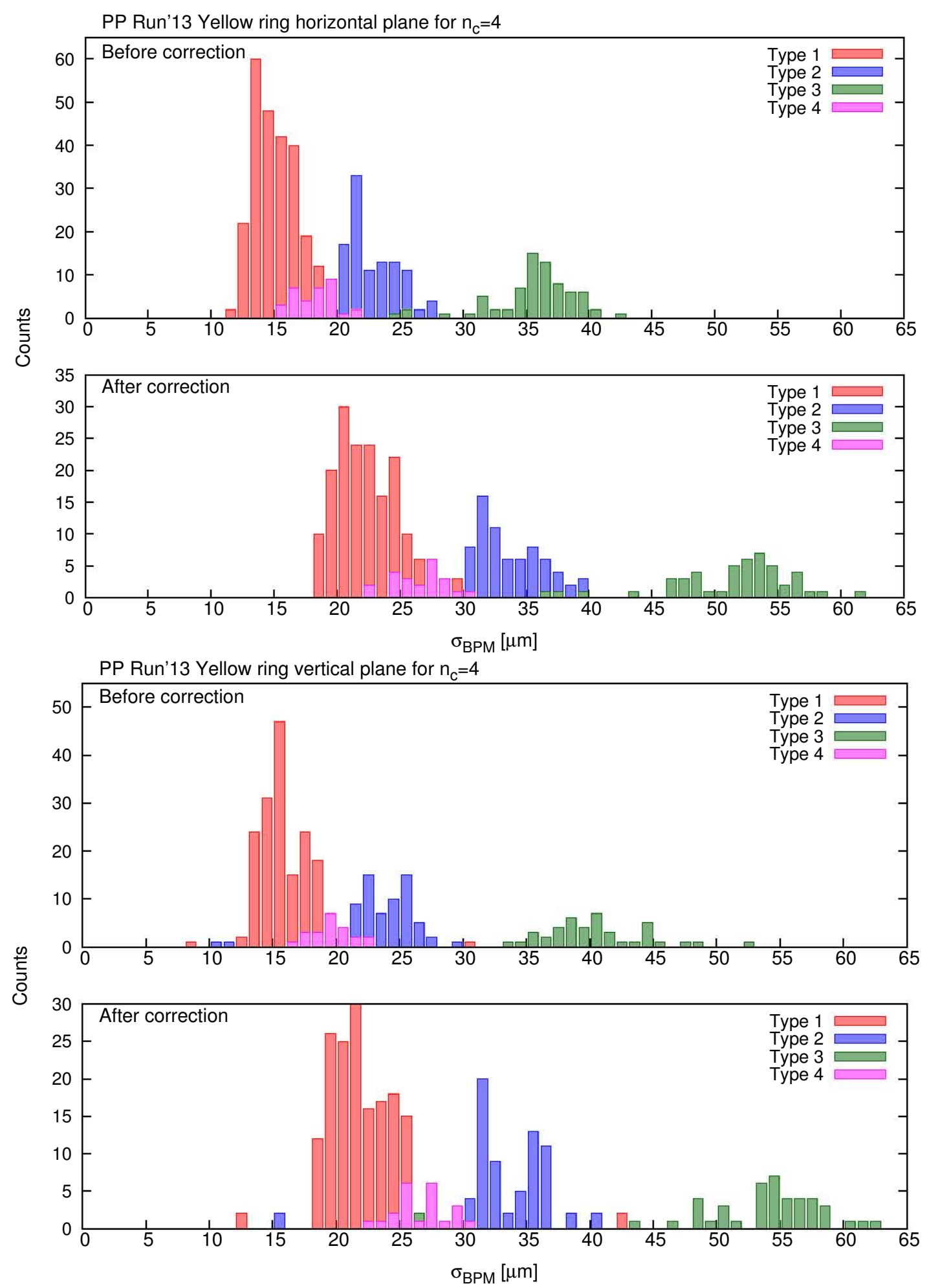

FIG. 9. Histogram of estimated noise levels of BPMs in the horizontal (top) and vertical (bottom) plane for the Yellow ring of PP Run'13 Fill\#17420 for $n_{c}=4$. The overall noise level becomes larger after the beta-beat correction. Roughly in each plot, $\sigma_{\text {type } 1} \approx \sigma_{\text {type } 2}<\sigma_{\text {type } 4}<\sigma_{\text {type } 3}<65 \mu$ m. 
TABLE I. Experimental parameters for PP Run'13 Fill\#17420.

\begin{tabular}{c|c|c|c|c}
\hline \hline \multirow{2}{*}{ Parameter } & \multicolumn{2}{|c|}{ Blue } & \multicolumn{2}{c}{ Yellow } \\
\cline { 2 - 5 } & Before correction & After correction & Before correction & After correction \\
\hline$\left(\epsilon_{x}, \epsilon_{y}\right)[\mu \mathrm{m} \cdot \mathrm{rad}]$ & $(19.42,15.61)$ & $(31.66,19.22)$ & $(12.75,9.45)$ & $(22.25,24.25)$ \\
\hline Bunch Intensity $\left[\times 10^{11}\right]$ & 1.39 & 1.38 & 1.50 & 1.318 \\
\hline Number of bunches & 6 & 6 & 7 & 7 \\
\hline AC dipole $\Delta \nu_{x, y}{ }^{\mathrm{a}}$ & \pm 0.008 & \pm 0.008 & \pm 0.008 & \pm 0.008 \\
\hline AC dipole $\Delta \theta_{x}(+,-)^{\mathrm{b}}[\mathrm{mrad}]$ & $(1.64,0.874)$ & $(1.07,1.18)$ & $(1.84,0.985)$ & $(1.13,0.970)$ \\
\hline AC dipole $\Delta \theta_{y}(+,-)^{\mathrm{c}}[\mathrm{mrad}]$ & $(1.04,1.81)$ & $(0.908,1.01)$ & $(1.04,1.86)$ & $(1.02,1.05)$ \\
\hline \hline
\end{tabular}

a $\Delta \nu_{x}$ and $\Delta \nu_{y}$ are the tune proximities for horizontal and vertical ac dipole to betatron tune, respectively.

b $\Delta \theta_{x}(+,-)$ are the maximum horizontal ac dipole kick amplitude for the plus and minus proximities.

c $\Delta \theta_{y}(+,-)$ are the maximum vertical ac dipole kick amplitude for the plus and minus proximities.

TABLE II. RHIC BPM mechanical details ${ }^{10}$

\begin{tabular}{ccccc}
\hline \hline BPM type & Warm/Cold & Number of Planes & Locations & Aperture $[\mathrm{mm}]$ \\
\hline 1 & cold & 1 & Q5, Q6, Q9, Q10-Q21 & 6.9088 \\
2 & cold & 2 & Q4, Q7, Q8 & 6.9088 \\
3 & cold & 2 & Q1, Q3, & 11.2725 \\
$4^{\mathrm{a}}$ & warm & 2 & DX, Dump, other warm & 11.2725 \\
\hline \hline
\end{tabular}

${ }^{a} \mathrm{In}^{10}$, this is classified as type 3 . For convenient it is marked as type 4 in this report.

as well as the not-optimized beam conditions. Beam intensity decreasd moderately as well after the correction.

Estimated BPM noise levels for the Blue and Yellow rings of the $\mathrm{CuAu}$ experiment with Fill number 16934 are shown in the top and bottom plots of Fig. 14, respectively. Similarly, the case with $n_{c}=6$ underestimates the noise at some DX BPMs. Histograms of noise level distribution for BPMs of the Blue and Yellow rings with $n_{c}=4$ are shown in the top and bottom plots of Fig. 15, respectively. In general, $\sigma_{\text {type } 1} \simeq \sigma_{\text {type } 2}$. At interaction point $2,6,8, \sigma_{\text {type } 3}>\sigma_{\text {type } 4}$, while in other locations, $\sigma_{\text {type } 3}<\sigma_{\text {type } 4}$. 

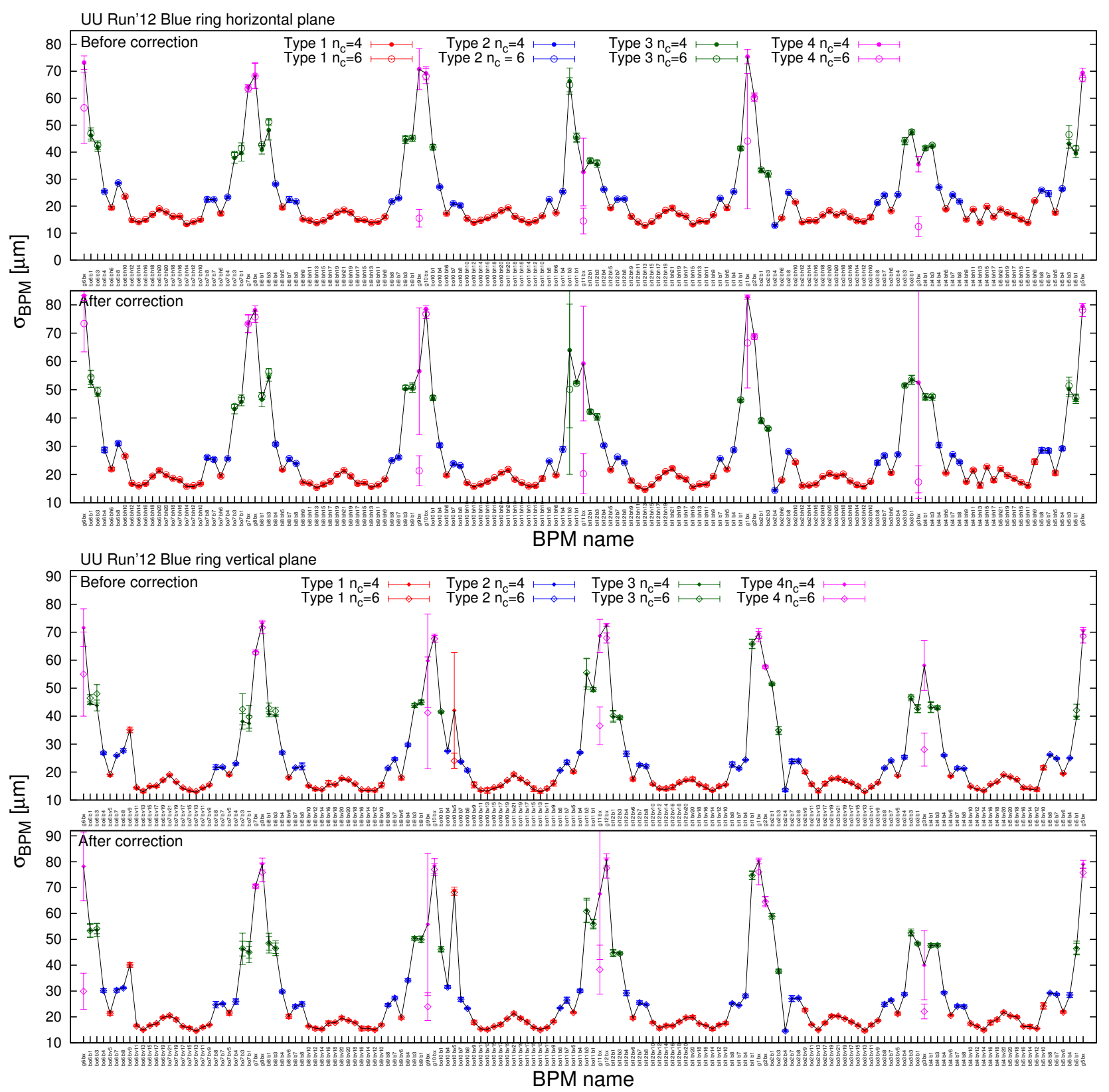

FIG. 10. Estimated noise levels of BPMs in the horizontal (top) and vertical (bottom) plane for the Blue ring of UU Run'12 Fill\#16814 for $n_{c}=4$ (solid points) and $n_{c}=6$ (hollow points). The case with $n_{c}=6$ underestimates the noise at some DX BPMs..

\section{v. CONCLUSION}

An ICA algorithm is applied for BPM noise level estimation from turn-by-turn data. The capability of the ICA algorithm for noise level estimation is validated by simulation. The ICA algorithm was applied to experimental turn-by-turn data from PP, UU, and $\mathrm{CuAu}$ experiments for noise level estimation. The Results show 4 types of characteristic noise level 

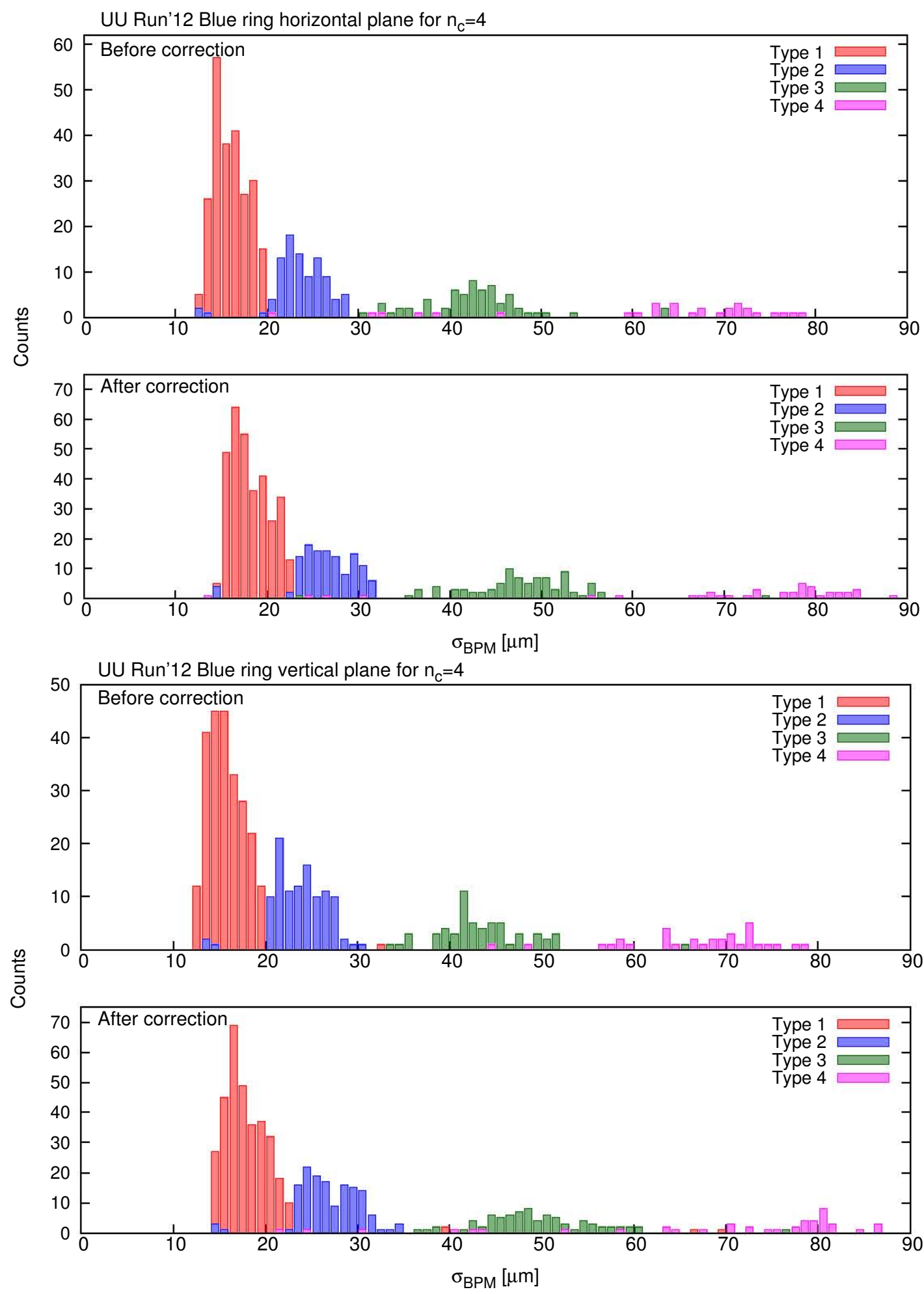

FIG. 11. Histogram of estimated noise levels of BPMs in the horizontal (top) and vertical (bottom) plane for the Blue ring of UU Run'12 Fill\#16814 for $n_{c}=4$. The overall noise level becomes larger after the beta-beat correction. Roughly in each plot, $\sigma_{\text {type } 1}<\sigma_{\text {type } 2}<\sigma_{\text {type } 3}<\sigma_{\text {type } 4}<90 \mu$ m. 

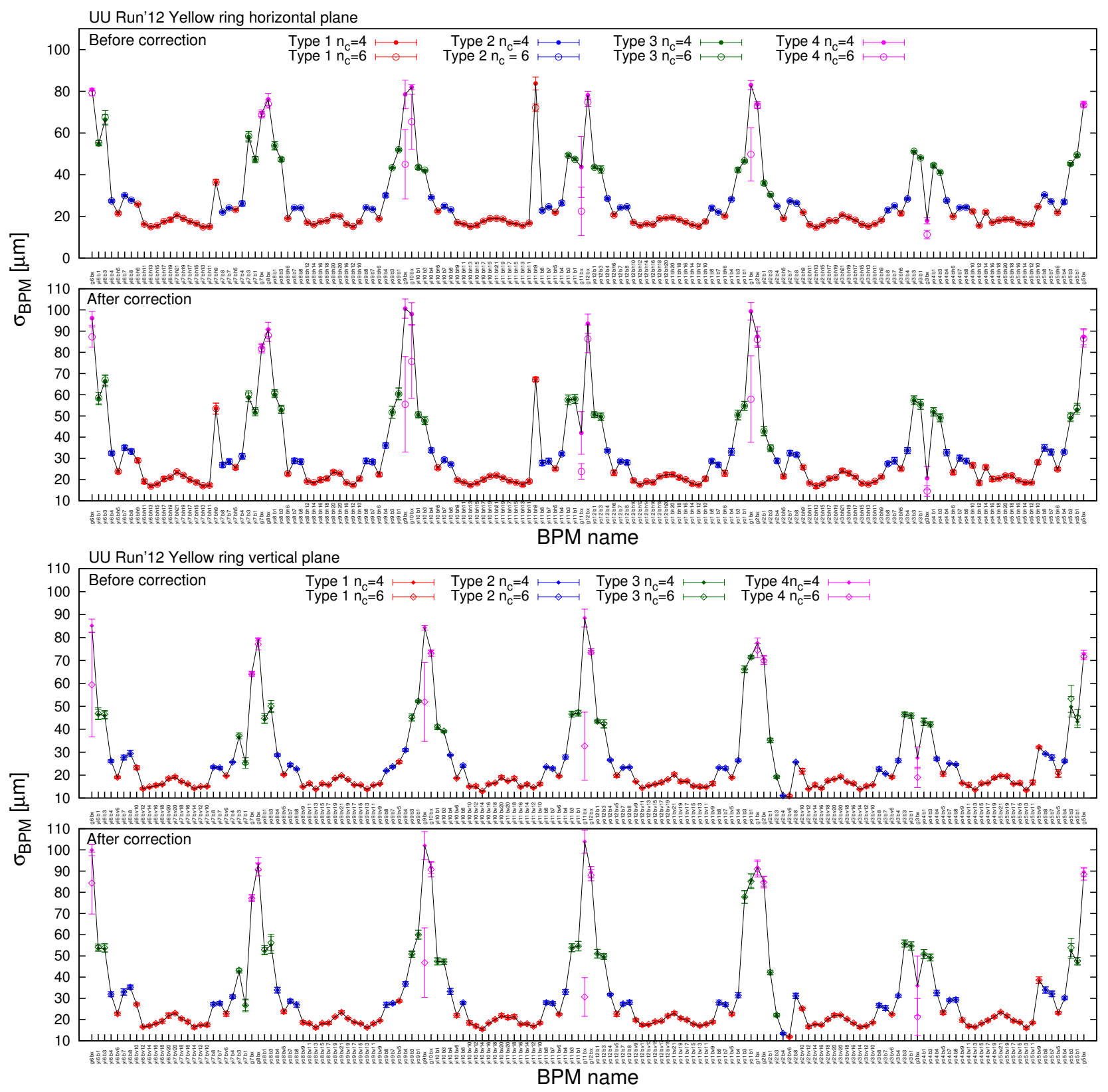

FIG. 12. Estimated noise levels of BPMs in the horizontal (top) and vertical (bottom) plane for the Yellow ring of UU Run'12 Fill\#16814 for $n_{c}=4$ (solid points) and $n_{c}=6$ (hollow points). The case with $n_{c}=6$ underestimates the noise at some DX BPMs.

distributions, which is consistent with the current configuration of 4 types of BPMs. A special pattern of noise level distribution was observed for the arc BPMs. DX BPMs shows quite different noise performance with respect to different ion species. Further investigations are needed to identify the sources of the noise level distribution. 

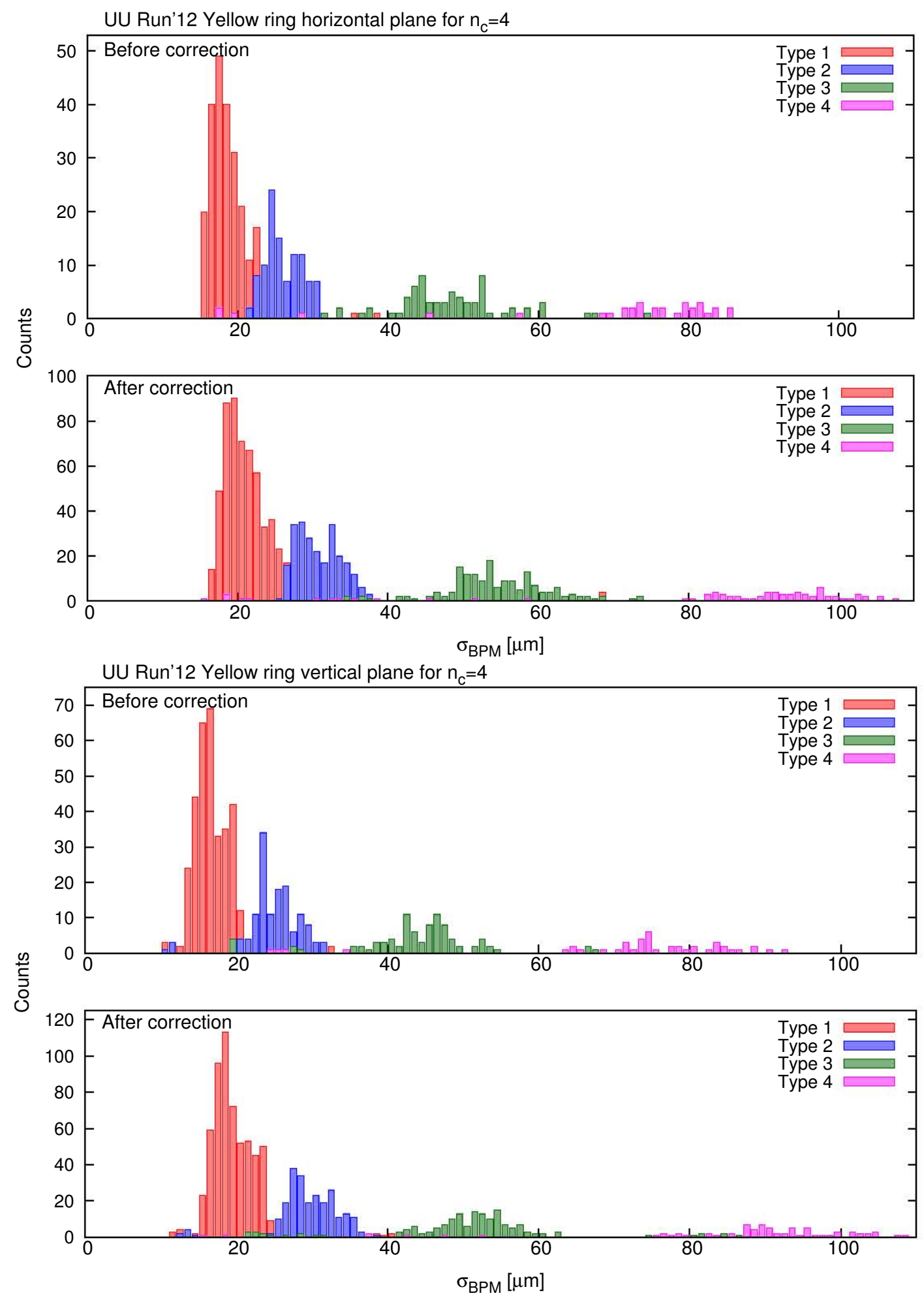

FIG. 13. Histogram of estimated noise levels of BPMs in the horizontal (top) and vertical (bottom) plane for the Yellow ring of UU Run'12 Fill\#16814 for $n_{c}=4$. The overall noise level becomes larger after the beta-beat correction. Roughly in each plot, $\sigma_{\text {type } 1}<\sigma_{\text {type } 2}<\sigma_{\text {type } 3}<\sigma_{\text {type } 4}<110 \mu$ m. 
TABLE III. Experimental parameters for UU Run'12 Fill\#16814

\begin{tabular}{c|c|c|c|c}
\hline \hline \multirow{2}{*}{ Experiment stage } & \multicolumn{2}{|c|}{ Blue } & \multicolumn{2}{c}{ Yellow } \\
\cline { 2 - 5 } & Before correction & After correction & Before correction & After correction \\
\hline$\left(\epsilon_{x}, \epsilon_{y}[\mu \mathrm{m} \cdot \mathrm{rad}]\right)$ & $(14.52,11.70)$ & $(29.87,28.27)$ & $(12.57,19.43)$ & $(17.38,26.34)$ \\
\hline Bunch Intensity $\left[\times 10^{11}\right]$ & 0.24 & 0.23 & 0.24 & 0.22 \\
\hline Number of bunches & 6 & 6 & 6 & 6 \\
\hline AC dipole $\Delta \nu_{x, y}{ }^{\text {a }}$ & 0.01 & 0.01 & 0.01 & 0.01 \\
\hline Hacd voltage $[\mathrm{V}]^{\mathrm{b}}$ & 0.2 & 0.2 & 0.2 & 0.2 \\
\hline Vacd voltage $[\mathrm{V}]^{\mathrm{c}}$ & 0.1 & 0.1 & 0.1 & 0.1 \\
\hline \hline
\end{tabular}

a Same as footnote a in Table. I.

b Maximum horizontal ac dipole kick in unit of volt.

${ }^{\mathrm{c}}$ Maximum vertical ac dipole kick in unit of volt.

TABLE IV. Experimental parameters for CuAu Run'12 Fill\#16814

\begin{tabular}{lcc}
\hline \hline Parameter & Blue & Yellow \\
\hline$\left(\epsilon_{x}, \epsilon_{y}\right)[\mu \mathrm{m} \cdot \mathrm{rad}]$ & $(28.63,15.76)$ & $(16.00,24.32)$ \\
Bunch Intensity $\left[\times 10^{11}\right]$ & 2.2 & 0.7 \\
Number of bunches & 13 & 12 \\
AC dipole $\Delta \nu_{x, y}{ }^{\mathrm{a}}$ & 0.01 & 0.01 \\
Hacd voltage $[\mathrm{V}]^{\mathrm{b}}$ & $0.1,0.2$ & 0.1 \\
Vacd voltage $[\mathrm{V}]^{\mathrm{c}}$ & 0.3 & 0.2 \\
\hline \hline
\end{tabular}

a Same as footnote a in Table III

b Same as footnote b in Table III

c Same as footnote c in Table III

${ }^{1}$ R. Calaga and R. Tomás, "Statistical analysis of rhic beam position monitors performance," Phys. Rev. ST Accel. Beams 7, 042801 (2004).

${ }^{2}$ A. Hyvarinen, J. Karhunen, and E. Oja, "Independent component analysis," STUDIES IN INFORMATICS AND CONTROL 11, 205-207 (2002).

${ }^{3}$ X. Huang, S. Lee, E. Prebys, and R. Tomlin, "Application of independent component analysis to fermilab booster," Physical Review Special Topics-Accelerators and Beams 8, 064001 (2005). 

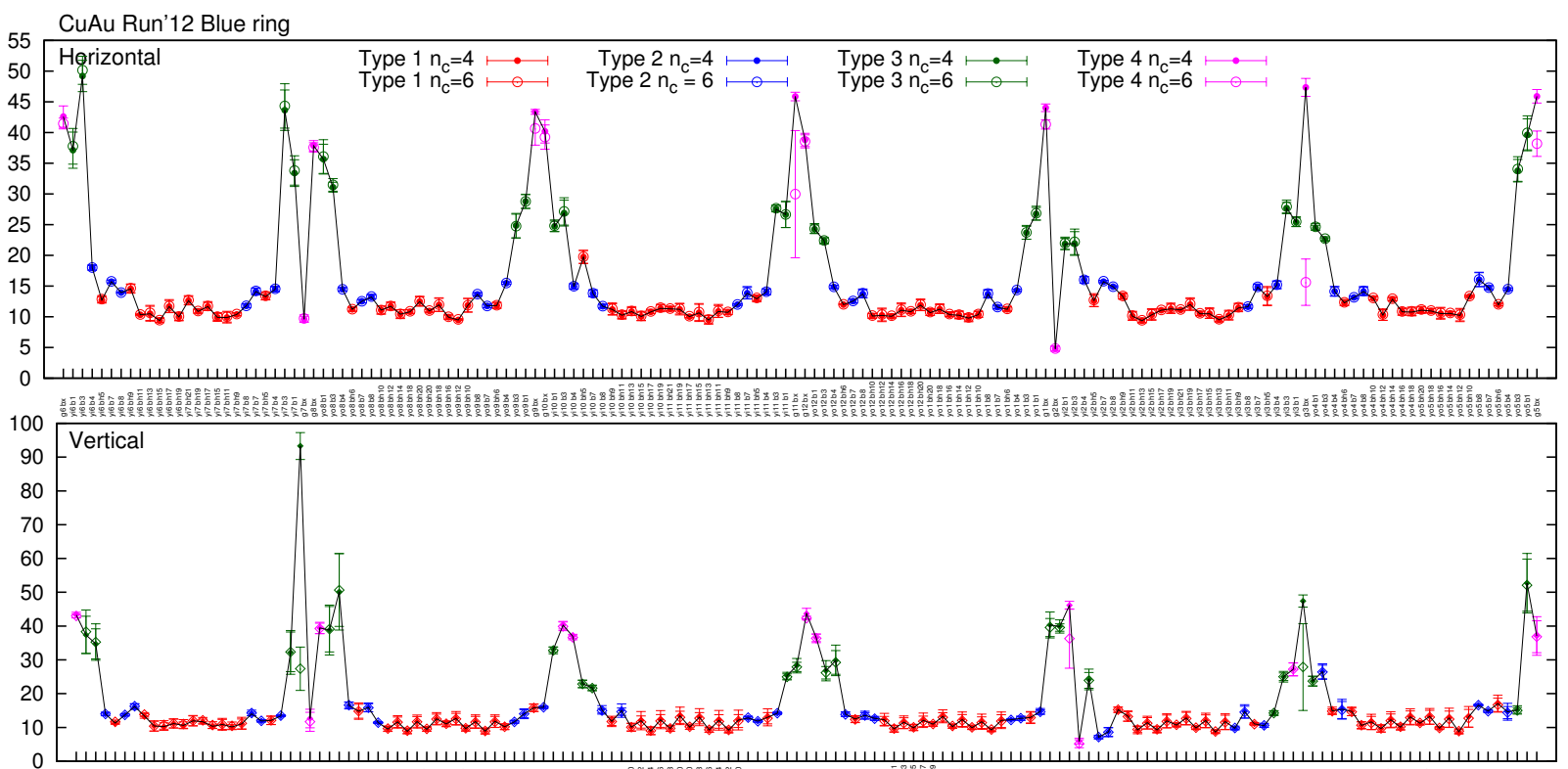

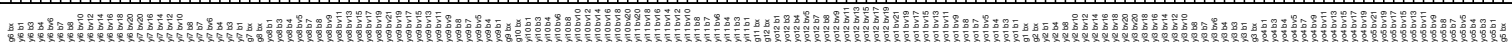

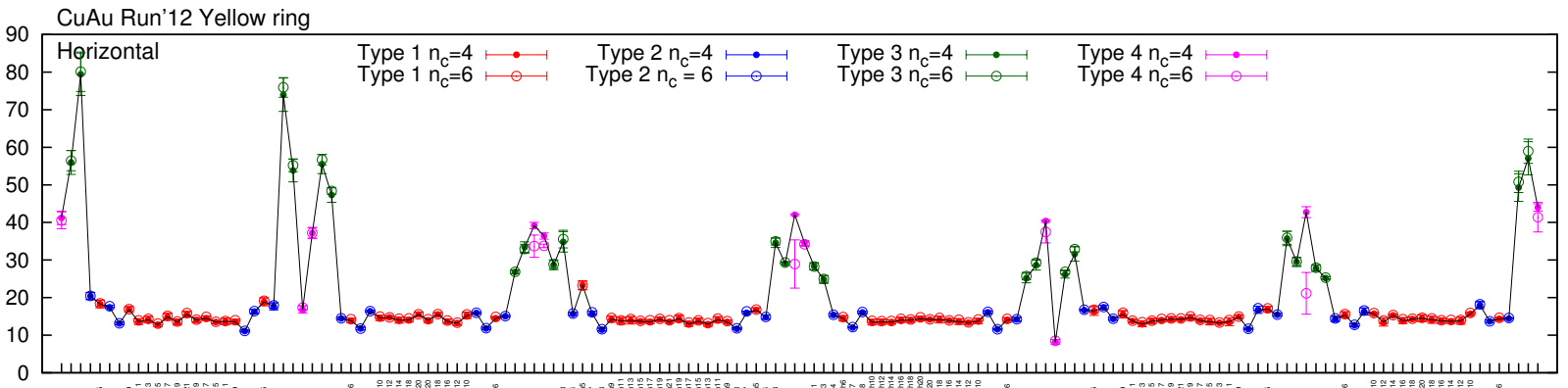

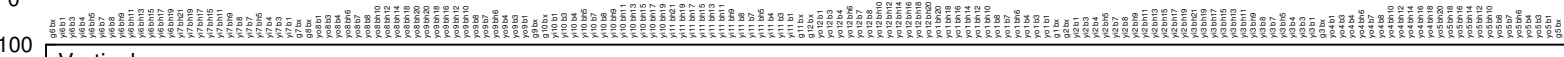

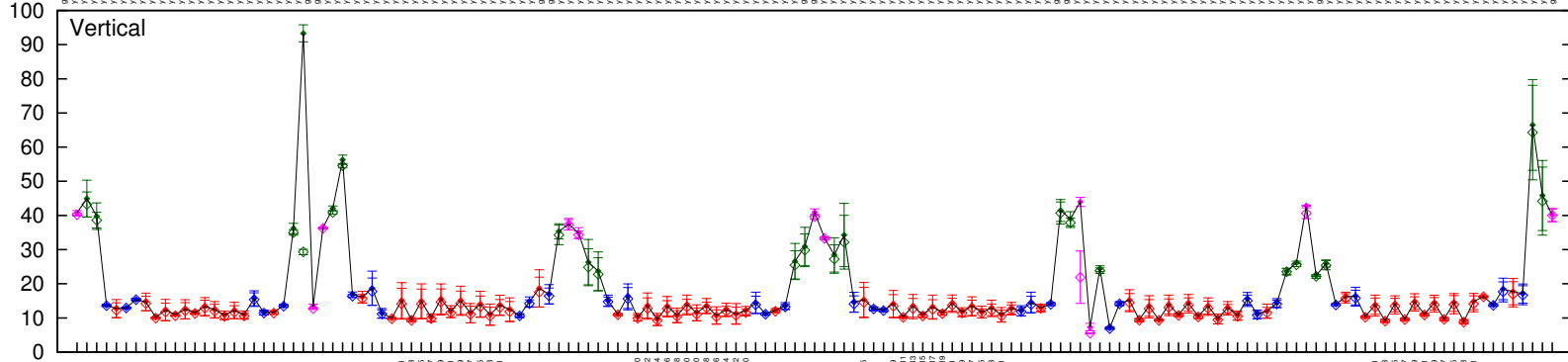

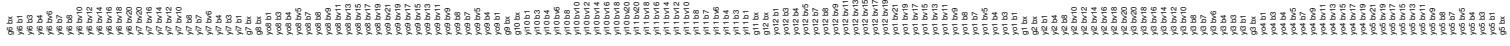

FIG. 14. Estimated noise levels of BPMs for the Blue ring (top) and Yellow ring (bottom) of $\mathrm{CuAu}$ Run'12 Fill\#16934 for $n_{c}=4$ (solid points) and $n_{c}=6$ (hollow points). The case with $n_{c}=6$ underestimates the noise at some DX BPMs. Generally, $\sigma_{\text {type } 1} \simeq \sigma_{\text {type } 2 \text {. At interaction point }}$ $2,6,8, \sigma_{\text {type } 3}>\sigma_{\text {type } 4}$, while in other locations, $\sigma_{\text {type } 3}<\sigma_{\text {type } 4}$.

${ }^{4}$ J. S. Kolski, R. J. Macek, R. C. McCrady, and X. Pang, "Independent component analysis applied to long bunch beams in the los alamos proton storage ring," Phys. Rev. ST Accel. Beams 15, 112802 (2012). ${ }^{5}$ X. Shen, S. Y. Lee, M. Bai, S. White, G. Robert-Demolaize, Y. Luo, A. Marusic, and R. Tomás, "Application of independent component analysis to ac dipole based optics measurement and correction at the 
CuAu Run'12 Blue ring horizontal plane for $n_{C}=4$
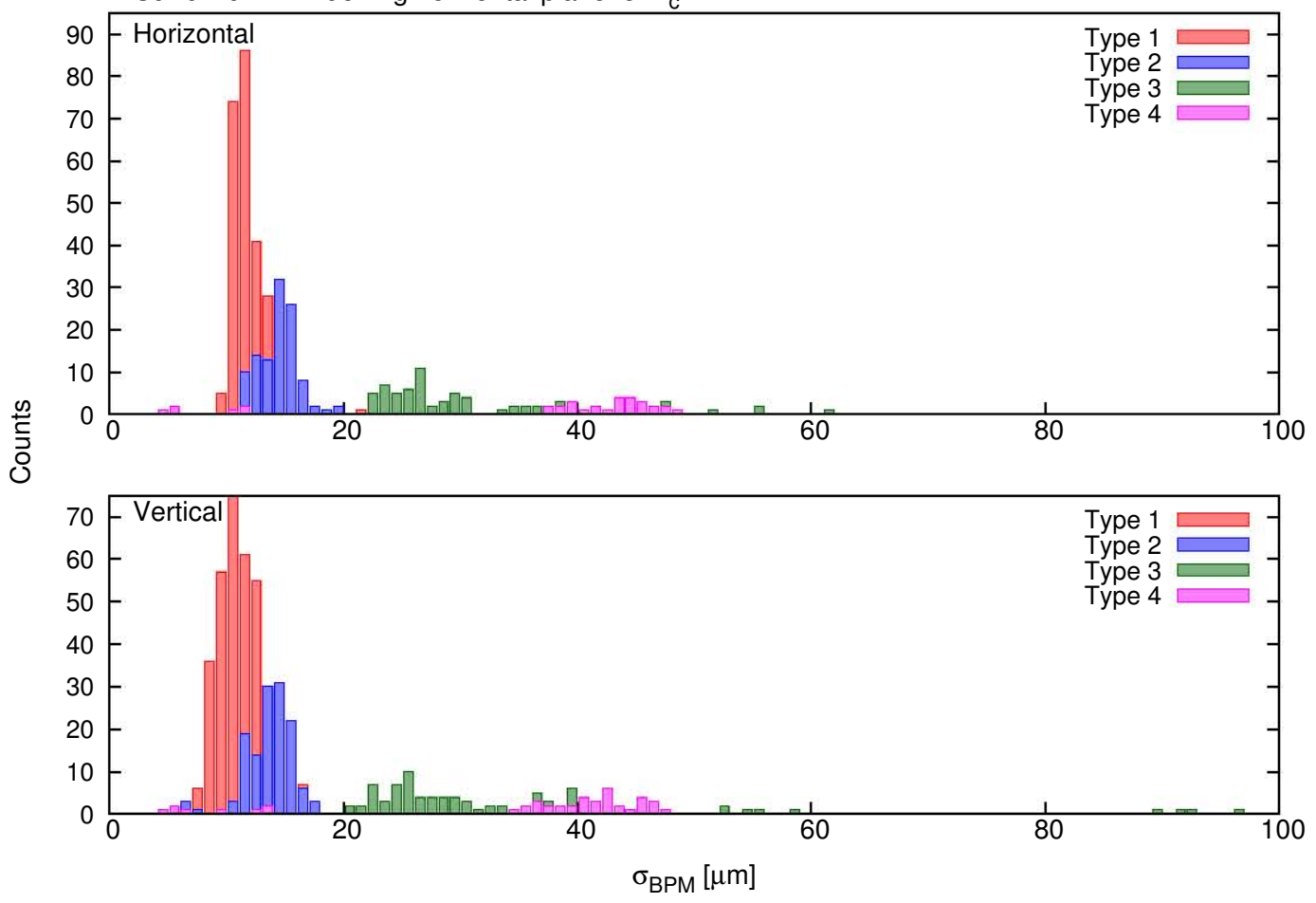

CuAu Run'12 Yellow ring horizontal plane for $n_{C}=4$
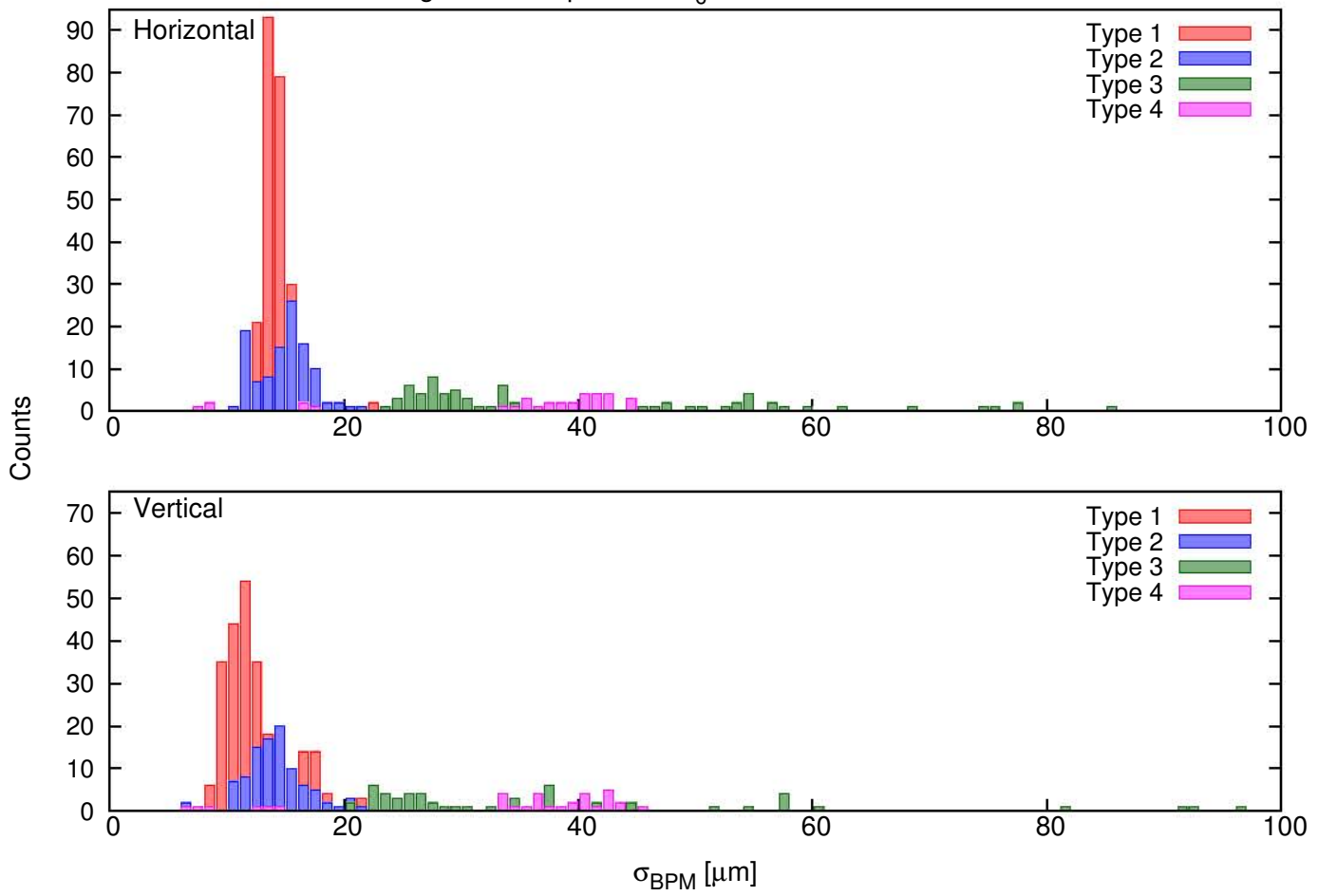

FIG. 15. Histogram of estimated noise levels of BPMs for the Blue ring (top) and Yellow ring (bottom) of CuAu Run'12 Fill\#16934. 
relativistic heavy ion collider," Phys. Rev. ST Accel. Beams 16, 111001 (2013).

${ }^{6}$ C.-x. Wang, V. Sajaev, and C.-Y. Yao, "Phase advance and $\beta$ function measurements using modelindependent analysis," Phys. Rev. ST Accel. Beams 6, 104001 (2003).

${ }^{7}$ M. Bai, S. Y. Lee, J. W. Glenn, H. Huang, L. Ratner, T. Roser, M. J. Syphers, and W. van Asselt, "Experimental test of coherent betatron resonance excitations," Phys. Rev. E 56, 6002-6007 (1997).

${ }^{8}$ J.-F. Cardoso, "Multidimensional independent component analysis," in Acoustics, Speech and Signal Processing, 1998. Proceedings of the 1998 IEEE International Conference on, Vol. 4 (IEEE, 1998) pp. 19411944.

${ }^{9}$ J.-F. Cardoso and A. Souloumiac, "Jacobi angles for simultaneous diagonalization," SIAM Journal on Matrix Analysis and Applications 17, 161-164 (1996).

${ }^{10}$ RHIC Instrumentation group, "RHIC BPM mechanical details," (2013).

${ }^{11}$ A. Marusic, personal communication. 